\title{
Synergetic Dispatch of Power System with Integration of Large-Scale Renewable Energy
}

\author{
Si Yang1, Zhongfu Jiang², Shan $\mathrm{Li}^{3}$, Wenbo Li ${ }^{4}$, Guojing Liuㄹ, Xiangyang Cao', Nan Wang1, \\ Lina Zhang1
}

${ }^{1}$ Economic \& Technology Research Institute, State Grid Shandong Electric Power Company, Jinan, China

${ }^{2}$ State Grid Shandong Electric Power Company, Jinan, China

${ }^{3}$ Key Laboratory of Power System Intelligent Dispatch and Control of Ministry of Education (Shandong University), Jinan, China

${ }^{4}$ State Grid Shandong Electric Power Research Institute, Jinan, China

${ }^{5}$ Economic \& Technology Research Institute, State Grid Jiangsu Electric Power Company, Nanjing, China

Email: yasi0408@163.com

How to cite this paper: Yang, S., Jiang, Z.F., Li, S., Li, W.B., Liu, G.J., Cao, X.Y., Wang, N. and Zhang, L.N. (2017) Synergetic Dispatch of Power System with Integration of Large-Scale Renewable Energy. Energy and Power Engineering, 9, 589-597. https://doi.org/10.4236/epe.2017.94B065

Received: November 30, 2016

Accepted: March 30, 2017

Published: April 6, 2017

\begin{abstract}
This paper comes up with a concept of synergetic advanced dispatch in order to deal with the ever-increasing uncertainty in power grid: Decision is made with respecting to AGC units and active load on the basis of synergetic unit combination such that active load's advantages in regulation speed is put to full use in achieving efficient cooperation with renewable energy power. Meanwhile, factoring in allowable frequency variation range during decisionmaking may help to reduce AGC units' regulation load and improve power grid's capacity of accommodating renewable energy power. Calculation example analysis suggested that the model and technique presented in this paper is capable of efficient coordination between active loads and renewable energy power, delivering friendly transition with day-ahead dispatch and AVC control.
\end{abstract}

\section{Keywords}

Power System, Synergetic Dispatch, Renewable Energy Generation, Frequency

\section{Introduction}

Advanced dispatch is a link that relates day-ahead dispatch to automatic generation control (AGC). Research on traditional power grid advanced dispatch is approaching to its mature stage at present [1]. With the integration of large scale of renewable energy power, however, the uncertainty faced by advanced dispatch becomes greater. The authors in [2] proposed the concept of allowable operation interval of renewable energy generation. References [3] build a dispatch model based on adjustable robust optimization and set an affine decision criteria be- 
tween adjustable variables and uncertain parameters by AGC unit participation factor. A multi-objective optimization model considering both the ability to resist disturbance and economic factors is built in [4] whose decision variables include AGC unit basis point and participation factor.

New technologies emerge in recent years, such as electric automobiles, energy storage, controllable load, and interruptible load, allowing changing their own generation or consumption power to a certain extent for what called active loads. Some researchers studied the control methods for active loads participating in primary [5] and secondary [6] frequency regulation of the grid as well as the decision-making method for active loads participating in unit combination [7] and dynamic economic dispatch with a $24 \mathrm{~h}$ anticipatory period [8]. Most of the researches have not considered active loads' role in reserve [7] [8], a few researches, though having considered that have not, dealt with how to make active load response decisions in advanced dispatch and how to attain intimate coordination with AGC unit reserve response. To cope with ever-increasing uncertainty, the reserve configuration of day-ahead dispatch should take into account the role played by active loads, the reserve response quantity of active loads shall be determined in advanced dispatch, and the AGC control should also consider the effect of active loads.

This paper studies synergetic advanced dispatch with integration of renewable energy. It makes decision for AGC unit and active load response on the basis of the result of synergetic unit combination such that active loads' advantage in regulation speed may be put to good use in coping with uncertainty associated with renewable energy power generation and conventional loads in order to realize an efficient cooperation. Additionally, the model has taken into account the fact that frequency variation is allowable within a certain limit, which has the effect of expanding the feasible region [9] and improving the grid's adoption capacity of renewable energy power.

\section{Description of Synergetic Advanced Dispatch Problem}

Synergetic dispatch of power grid requires intimate coordination of decisionmaking at various time scale such as day-ahead level (unit combination), advanced level, and real time level, among which, synergetic advanced dispatch is the link that relates day-ahead dispatch to AGC control.

\subsection{Correlation with Synergetic Unit Combination}

With non-AGC units, in synergetic dispatch, it is AGC units and active loads that take up reserve and the capability is therefore greater in coping with uncertainties; because of this, synergetic advanced dispatch decision-making no longer considers the re-decision-making of non-AGC unit output power, so that non-AGC units are operated according to the power generation schedule specified in day-ahead dispatch.

With AGC units, synergetic unit combination configuration specifies reserve capacity whereby AGC units may cope with uncertainties in advanced time level. 
In synergetic advanced dispatch, the reserve response quantity of AGC units shall be determined according to super-short period prediction outcomes of renewable energy power generation and conventional loads.

With active loads, synergetic unit combination is a process that allocates this finite dispatch resource and develops a power storage schedule, depending on daily periodic pattern of renewable energy power generation and conventional loads. The conflict shall be avoided that decision-making in synergetic advanced dispatch is contradictory with synergetic unit combination, otherwise the power demand by active loads might not be satisfied.

\subsection{Correlation with Synergetic AGC Control}

Synergetic advanced dispatch provides a base point which may be used by AGC units and by active loads while participating in AGC control, and dispatch errors as well as power fluctuations occurring in the dispatch period need to be balanced by AGC control.

According to [6], assuming the regulation quantity demand, arising from the discrepancy between the actual power and the expected power, is $\Delta P_{t}$ in period $t$ during synergetic advanced dispatch, then the adjustment quantity $\Delta P_{A, t}$ of active loads can be written as:

$$
\Delta P_{A, t}=\left\{\begin{array}{lll}
\Delta P_{t} & \text { if } & \left|\Delta P_{t}\right| \leq\left|\Delta P_{A, t}^{\max }\right| \\
\Delta p_{A, t}^{\max } & \text { if } & \left|\Delta P_{t}\right| \geq\left|\Delta P_{A, t}^{\max }\right|
\end{array}\right.
$$

where, $\Delta P_{A, t}^{\max }$ is the maximum regulation capacity of active loads in time period $t$.

Then, the adjustment quantity $\Delta P_{\mathrm{AGC}, t}$ of AGC units can be expressed as below:

$$
\Delta P_{A G C, t}= \begin{cases}\Delta P_{t}-\Delta P_{A, t} & \text { if }\left|\Delta P_{t}-\Delta P_{A, t}\right| \leq\left|\Delta P_{A G C, t}^{\max }\right| \\ \Delta P_{A G C, t}^{\max } & \text { if }\left|\Delta P_{t}-\Delta P_{A, t}\right| \geq\left|\Delta P_{A G C, t}^{\max }\right|\end{cases}
$$

where, $\Delta P_{A G C, t}^{\max }$ is the maximum regulation capacity of AGC units in time period $t$.

After regulation of active loads and AGC unit, if there is:

$$
\Delta P_{t}-\Delta P_{A, t}-\Delta P_{A G C, t}>0
$$

Suppose a system frequency regulation coefficient of $\beta_{t}$ at time period $t$, when Equation (3) is valid the power grid frequency deviation $\Delta f_{t}$ is:

$$
\Delta f_{t}=\frac{\Delta P_{t}-\Delta P_{A, t}-\Delta P_{A G C, t}}{\beta_{t}}
$$

Rejection of renewable energy power or switching off loads becomes necessary only when $\Delta f_{t}$ is out of the allowable frequency scope.

\section{Synergetic Advanced Dispatch Model}

\subsection{Model Description}

Assuming $\Omega_{R}$ and $\Omega_{D}$ are uncertainty sets corresponding to renewable energy 
power generation and conventional loads, then the power $p_{R}$ of renewable energy power generation and the power $p_{D}$ of conventional loads shall satisfy:

$$
\left\{\begin{array}{l}
p_{R} \in \Omega_{R} \\
p_{D} \in \Omega_{D}
\end{array}\right.
$$

Advanced dispatch decision-making outcomes shall satisfy the uncertainty set of renewable energy power generation and conventional loads so that it is modeled as below:

$$
\begin{gathered}
\min f\left(u, y, \Omega_{R}, \Omega_{D}\right) \\
\left\{\begin{array}{l}
h\left(u, y\left(p_{R}, p_{D}\right), p_{R}, p_{D}\right)=0 \\
g\left(u, y\left(p_{R}, p_{D}\right), p_{R}, p_{D}\right) \leq 0 \quad \forall p_{R} \in \Omega_{R} \\
\underline{u} \leq u \leq \bar{u} \\
\underline{y} \leq y\left(p_{R}, p_{D}\right) \leq \bar{y}
\end{array} \quad \forall p_{D} \in \Omega_{D}\right.
\end{gathered}
$$

where, in (6) and (7), $f$ is the cost function; $u$ is the control quantity needing advanced decision; $y$ is a buffer quantity designed to cope with potential fluctuations; $h$ is the equality constraints, $g$ is the inequality constraints, $\bar{u}$ and $\underline{u}$ are the maximum and minimum regulation scope limits of control quantity respectively; $\bar{y}$ and $\underline{y}$ are the maximum and minimum regulation scope limits of buffer quantity respectively.

\subsection{Mathematical Model}

With minimization of AGC units' operation cost and rejected renewable energy power as the objective, the objective function of this model may be written as:

$$
\min \sum_{t=1}^{T} \sum_{i=1}^{N}\left(a_{i} P_{G, i, t}^{2}+b_{i} P_{G, i, t}+c_{i}\right)+\sum_{t=1}^{T} \gamma_{t}\left(P_{R, t}^{\max }-\bar{P}_{R, t}\right)
$$

where, $T$ is the number of time periods, $N$ is the number of AGC units, $P_{G, i, t}$ is the base point of AGC unit $i$ at time period $t, a_{i}, b_{i}$ and $c_{i}$ are characteristic coefficients of AGC unit $i$.

Suppose that the predicted interval of renewable energy power generation for time period $t$ is $\left[P_{R, t}^{\min }, P_{R, t}^{\max }\right]$. If renewable energy power generation interval in synergetic advanced dispatch is $\left[\underline{P}_{R, t}, \bar{P}_{R, t}\right]$, where $\underline{P}_{R, t}$ and $\bar{P}_{R, t}$ are decision quantities, then the rejected renewable energy power is written as $P_{R, t}^{\min }-\bar{P}_{R, t}$, with $\gamma_{t}$ being the penalty coefficient.

Constraints mainly include:

1) Base point power equilibrium constraint

$$
P_{N A G C, t}^{*}+\sum_{i=1}^{N} P_{G, i, t}+P_{R, t}=D_{t}+P_{A, t}
$$

where, $P_{N A G C, t}^{*}$ is the sum of output power of non-AGC units in time period $t$; $P_{R, t}$ is scheduled renewable energy power generation value in time period $t$ and is a decision quantity; $D_{t}$ is the super short period power prediction expected value of conventional load in time period $t, P_{A, t}$ is the charge/discharge power of active loads in time period $t$ and is also a decision quantity, with $P_{A, t}>0$ indicating the 
active loads are being charged.

2) Operational constraints of active loads

$$
\begin{gathered}
-P_{A, t}^{\max } \leq P_{A, t} \leq P_{A, t}^{\max } \\
E_{t}=E_{t-1}+P_{A, t} \cdot \Delta t \\
E_{t}^{\min } \leq E_{t} \leq E_{t}^{\max } \\
E_{T}=E^{e n d}
\end{gathered}
$$

where, $P_{A, t}^{\max }$ is the maximum charge power allowable in time period $t$ and is assumed to be equal to the peak discharge power; $E_{t}$ is the energy stored by active loads in time period $t, E_{t}^{\max }$ and $E_{t}^{\min }$ are the maximum and minimum stored energy allowed in time period $t, E^{\text {end }}$ is the expected stored energy at the end of dispatch.

3) Coping with uncertainty constraints

Supposing conventional load distribution interval is $\left[\underline{P}_{D, t}, \bar{P}_{D, t}\right]$ in time period $t$, it is straightforward to find the net load distribution interval $\left[\underline{P}_{N, t}, \bar{P}_{N, t}\right]$ in time period $t$, with

$$
\left\{\begin{array}{l}
\bar{P}_{N, t}=\bar{P}_{D, t}-\underline{P}_{R, t} \\
\underline{P}_{N, t}=\underline{P}_{D, t}-\bar{P}_{R, t}
\end{array}\right.
$$

where, $D_{t}-P_{R, t}$ in (9) is the reference value $P_{N, t}$ of net load in time period $t$.

An illustration is provided below using the upper boundary $\bar{P}_{N, t}$ of the net load distribution interval as an example. In this case, the adjustment quantity is $\bar{P}_{N, t}-P_{N, t}$.

In the case of active loads, the actual adjustment quantity $\bar{P}_{A, t}$ taken up by active loads may be written as:

$$
\bar{P}_{A, t}=\left\{\begin{array}{l}
\bar{P}_{N, t}-P_{N, t} \quad \text { if }\left(\bar{P}_{N, t}-P_{N, t}\right) \leq\left(P_{A, t}+P_{A, t}^{\max }\right) \\
P_{A, t}+P_{A, t}^{\max } \quad \text { if }\left(\bar{P}_{N, t}-P_{N, t}\right) \geq\left(P_{A, t}+P_{A, t}^{\max }\right)
\end{array}\right.
$$

with the rest of adjustment quantity demand being $\bar{P}_{N, t}-P_{N, t}-\bar{P}_{A, t}$ and the adjustment quantity corresponding to AGC unit $i$ is:

$$
\bar{P}_{G, i, t}=\rho_{G, i, t}\left(\bar{P}_{N, t}-P_{N, t}-\bar{P}_{A, t}-\beta_{t} \cdot \Delta \underline{f_{t}}\right)
$$

where, $\rho_{G, i, t}$ is the participation factor of AGC unit $i$ in time period $t$.

Similarly, when the net load drops to the lower boundary $\underline{P}_{N, t}$, there are:

$$
\begin{gathered}
\underline{P}_{A, t}= \begin{cases}P_{N, t}-\underline{P}_{N, t} & \text { if }\left(P_{N, t}-\underline{P}_{N, t}\right) \leq\left(P_{A, t}^{\max }-P_{A, t}\right) \\
P_{A, t}^{\max }-P_{A, t} & \text { if }\left(P_{N, t}-\underline{P}_{N, t}\right) \geq\left(P_{A, t}^{\max }-P_{A, t}\right)\end{cases} \\
\underline{P}_{G, i, t}=\rho_{G, i, t}\left(P_{N, t}-\underline{P}_{N, t}-\underline{P}_{A, t}-\beta_{t} \cdot \Delta \overline{f_{t}}\right)
\end{gathered}
$$

4) Upper and lower limit constraints of AGC unit output power:

$$
\begin{aligned}
& P_{G, i, t}^{\min } \leq P_{G, i, t}+\bar{P}_{G, i, t} \leq P_{G, i, t}^{\max } \\
& P_{G, i, t}^{\min } \leq P_{G, i, t}-\underline{P}_{G, i, t} \leq P_{G, i, t}^{\max }
\end{aligned}
$$

where, $P_{G, i, t}^{\max }$ and $P_{G, i, t}^{\min }$ are the upper and lower boundary of AGC unit $i$ out- 
put power.

5) Constraint of AGC unit creeping speed:

$$
\begin{aligned}
& \left(P_{G, i, t}+\bar{P}_{G, i, t}\right)-\left(P_{G, i, t-1}-\underline{P}_{G, i, t-1}\right) \leq r_{G, i} \cdot \Delta t \\
& \left(P_{G, i, t-1}+\bar{P}_{G, i, t-1}\right)-\left(P_{G, i, t}-\underline{P}_{G, i, t}\right) \leq r_{G, i} \cdot \Delta t
\end{aligned}
$$

where, $r_{G, i}$ is the maximum adjustment speed of unit $i, \Delta t$ being the duration of time.

6) Constraint of frequency deviation:

$$
0 \leq \Delta \underline{f_{t}}, \Delta \bar{f}_{t} \leq \Delta f^{\max }
$$

where, $\Delta f^{\max }$ is the maximum allowable frequency deviation for power grid operation.

7) Constraint of renewable energy power generation operation interval:

$$
\begin{aligned}
& 0 \leq \bar{P}_{R, t} \leq P_{R, t}^{\max } \\
& 0 \leq \underline{P}_{R, t} \leq P_{R, t}^{\min } \\
& \underline{P}_{R, t} \leq P_{R, t} \leq \bar{P}_{R, t}
\end{aligned}
$$

8) Constraint of AGC unit participation factors:

$$
\sum_{i=1}^{N} \rho_{G, i, t}=1
$$

\subsection{Solution Strategy}

In the model above, Equation (8) is in quadratic form, Equations (15) and (17) are logical expressions, and Equations (16) and (18) contain nonlinear expression of the product of participation factors. To deal with that, the following measures are taken:

Firstly, the quadratic objective function is piecewise linearized.

Secondly, logical expressions (15) and (17) are converted into a general form by introducing $0-1$ auxiliary variables. For (15), $0-1$ quantity $\bar{u}_{t}$ is introduced:

$$
\begin{gathered}
\frac{\left(P_{A, t}+P_{A, t}^{\max }\right)-\left(\bar{P}_{N, t}-P_{N, t}\right)}{M} \leq \bar{u}_{t} \leq 1+\frac{\left(P_{A, t}+P_{A, t}^{\max }\right)-\left(\bar{P}_{N, t}-P_{N, t}\right)}{M} \\
-\left(1-\bar{u}_{t}\right) M \leq \bar{P}_{A, t}-\left(\bar{P}_{N, t}-P_{N, t}\right) \leq 0 \\
-\bar{u}_{t} M \leq \bar{P}_{A, t}-\left(P_{A, t}+P_{A, t}^{\max }\right) \leq 0
\end{gathered}
$$

where, $M$ is a constant with a high value and $\bar{u}_{t}$ is 1 if $\left(P_{A, t}+P_{A, t}^{\max }\right)>\left(\bar{P}_{N, t}-P_{N, t}\right)$ or 0 if $\left(P_{A, t}+P_{A, t}^{\max }\right)<\left(\bar{P}_{N, t}-P_{N, t}\right)$.

Analysis discovers that (15) is equivalent to (28)-(30). Similarly, logical expression (17) can be converted to a general form consisted of (31)-(33).

$$
\begin{gathered}
\frac{\left(P_{A, t}^{\max }-P_{A, t}\right)-\left(P_{N, t}-\underline{P}_{N, t}\right)}{M} \leq \underline{u}_{t} \leq 1+\frac{\left(P_{A, t}^{\max }-P_{A, t}\right)-\left(P_{N, t}-\underline{P}_{N, t}\right)}{M} \\
-\left(1-\underline{u}_{t}\right) M \leq \underline{P}_{A, t}-\left(P_{N, t}-\underline{P}_{N, t}\right) \leq 0 \\
-\underline{u}_{t} M \leq \underline{P}_{A, t}-\left(P_{A, t}^{\max }-P_{A, t}\right) \leq 0
\end{gathered}
$$


Finally, AGC unit participation factors are taken as constant using the following equation in this paper.

$$
\rho_{G, i, t}=1 /\left[a_{i} \times\left(\sum_{j=1}^{N} 1 / a_{j}\right)\right]
$$

\section{Analysis of Calculation Example}

\subsection{Description of Calculation Example}

The model described above was simulated using a 10-unit system in which unit $1-6$ are supposed to be AGC units and units parameters, load curve and day-ahead dispatch results are given by [10]. Batteries were included by way of example of active loads, the peak charge/discharge power is $50 \mathrm{MW}$ and the maximum and minimum values of last time period energy are $110 \mathrm{MWh}$ and $70 \mathrm{MWh}$. Renewable energy power generation is consisted mainly of wind power and its prediction result is in Figure 1. The system frequency was 50 $\mathrm{Hz}$, with an allowable variation range of $50 \pm 0.1 \mathrm{~Hz}$ for normal operation. $1 \%$ variation in system frequency gave rise to $2.89 \%$ variation in active power of conventional loads.

\subsection{Conventional Advanced Dispatch Computation Results}

Suppose that day-ahead dispatch sets active load power at $25 \mathrm{MW}$ and traditional advanced dispatch does not consider the adjustment for active load so that the charging power is treated as a fixed constant in decision-making. With traditional dispatch, the following figure compares the wind power operation interval as predicted and the operation interval as actually established in decision-making.

As is seen from Figure 1, with traditional advanced dispatch, one has to reject wind power when wind power is associated with higher uncertainty, thus unable to satisfy the maximum operation interval predicted.

\subsection{Synergetic Advanced Dispatch Computation Results}

The model and computation method proposed in this paper, in contrast, have

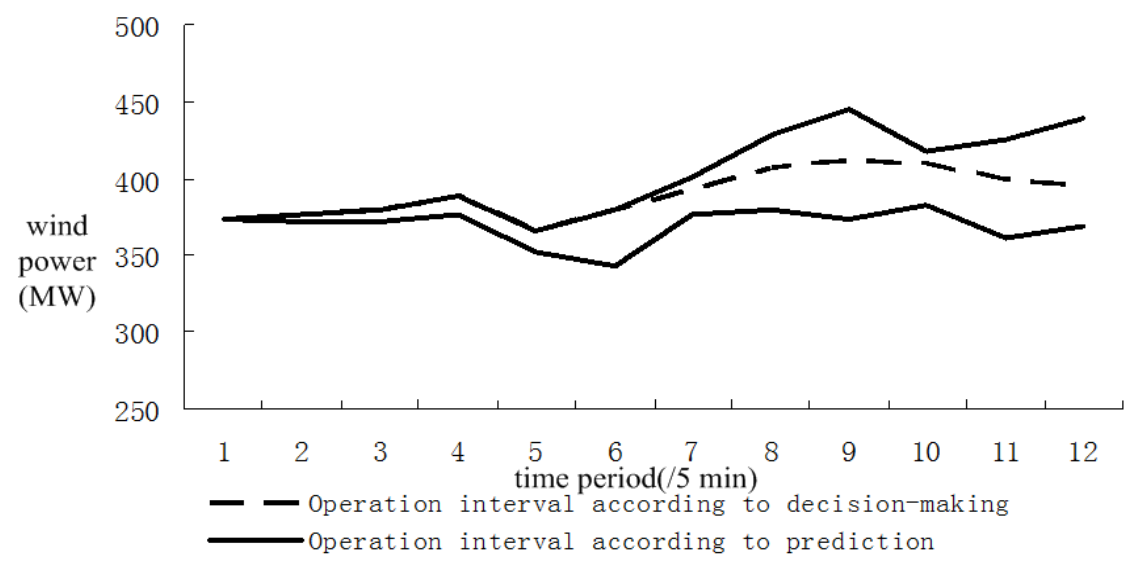

Figure 1. Wind power operation interval in conventional dispatch. 
factored in active load reserve response and frequency regulation effect, so it is no longer needed to reject wind power in this example, hence completely satisfying the predicted wind power operation interval.

To validate dispatch outcomes, Monte Carlo simulation was performed during which wind power output power values were generated randomly to simulate actual charge/discharge power of active loads and variation in power grid frequency.

As is evident from Figure 2, with synergetic advanced dispatch, active load charge/discharge power is no longer locked but is determined by the uncertainty of wind power. Relative to locking active load charge/discharge power used in conventional dispatch, this approach is more likely to produce efficient cooperation between the two.

In the time period when active loads are unable to satisfy power uncertainty, active power equilibrium may be achieved by readjusting the AGC unit output power or by frequency regulation effect. If in AGC control, the objective is to reduce AGC unit regulation to a minimum and let power fluctuation be preferably taken up by frequency regulation effect, then the simulated frequency is as shown in Figure 3. In time periods with higher uncertainty, the frequency

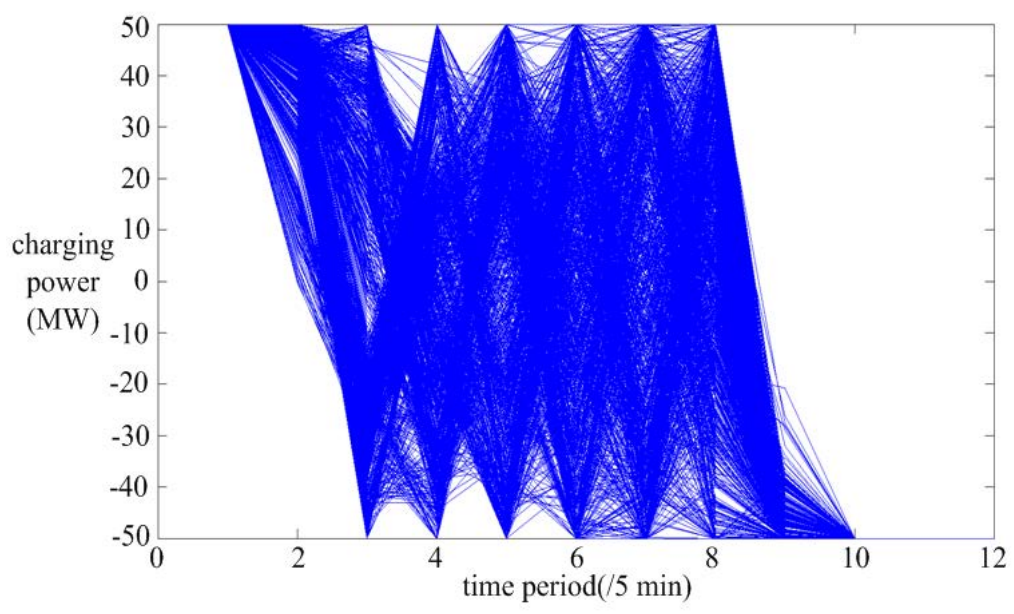

Figure 2. Simulation results of active load charge/discharge conditions.

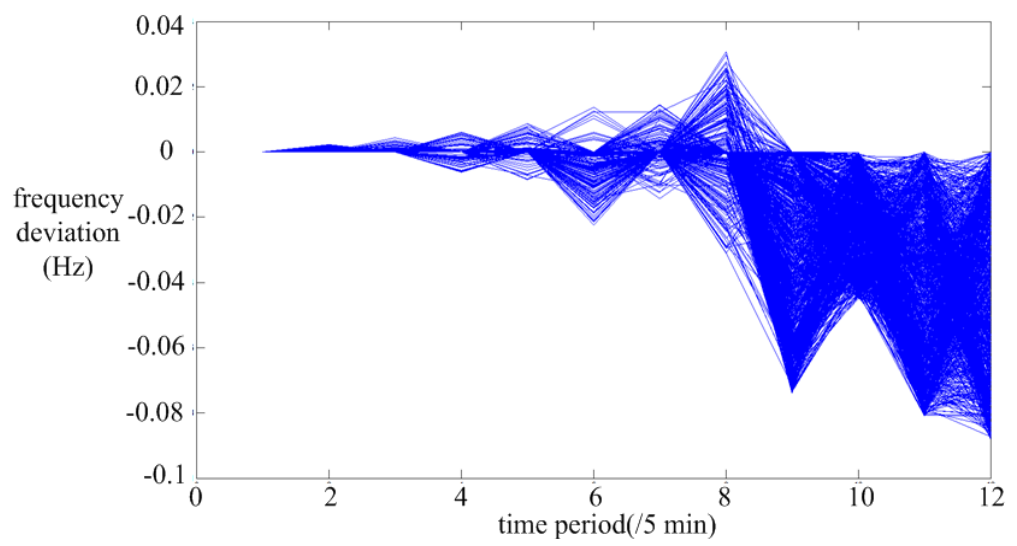

Figure 3. Simulation Results of power grid frequency deviation. 
deviation is larger, but remains within the allowable deviation range.

\section{Conclusion}

This study investigated synergetic advanced dispatch, coming to the following major conclusions: 1) Coordination with reserve configuration in synergetic unit combination and factoring in adjusting effect of active loads are able to make full use of active load's advantages in regulation speed and achieve more cooperation with renewable energy power generation; 2) Consideration of active loads and frequency regulation effect in advanced dispatch model is conducive to alleviate AGC units' regulation pressure in the control process and avoid conservative decision; 3) Synergetic advanced dispatch realizes friendly transition from dayahead dispatch to AGC control and improves power grid's adaptation to renewable energy power.

\section{References}

[1] Innorta, M., Marannino, P., Granelli, G. and Montagna, M. (1988) Security Constrained Dynamic Dispatch of Real power for Thermal Groups. IEEE Transactions on Power Systems, 3, 774-781. https://doi.org/10.1109/59.192934

[2] Zhao, J., Zheng, T. and Litvinov, E. (2014) Variable Resource Dispatch through Do-not-exceed Limit. IEEE Transactions on Power Systems, 30, 1-9.

[3] Jabr, R.A. (2013) Adjustable Robust OPF with Renewable Energy Sources. IEEE Transactions on Power Systems, 28, 4742-4751. https://doi.org/10.1109/TPWRS.2013.2275013

[4] Yang, M., Cheng, F.L. and Han, X.S. (2015) Real-time Dispatch Based on Effective Steady-state Security Regions of Power System. Proceedings of the CSEE, 35, 1353-1362.

[5] Liu, H., Hu, Z., Song Y. and Lin, J. (2013) Decentralized Vehicle-to-Grid Control for Primary Frequency Regulation Considering Charging Demands. IEEE Transactions on Power Systems, 28, 3480-3489. https://doi.org/10.1109/TPWRS.2013.2252029

[6] Cheng, Y., Tabrizi, M., Sahni, M. and Povedano, A. (2014) Dynamic Available AGC Based Approach for Enhancing Utility Scale Energy Storage Performance. Smart Grid IEEE Transactions on, 5, 1070-1078. https://doi.org/10.1109/TSG.2013.2289380

[7] Xie,Y.G. and Jiang, X.D. (2011) Impact of Energy Storage System on the Unit Commitment Problem with Volatile Wind Power. Automation of electric power systems, 35, 19-24.

[8] Zhao, J.H., Wen, F.S., Xue, Y.S, Dong, Z.Y. and Xin, J.B. (2010) Power System Stochastic Economic Dispatch Considering Uncertain Outputs from Plug-in Electric Vehicles and Wind Generators. Automation of Electric Power Systems, 34, 22-29.

[9] Liu, G.J., Han, X.S. and Yang, M. (2014) Synergetic Economic Dispatch in Power System Operation. Proceedings of the CSEE, 34, 2668-2675.

[10] Han, X.S. and Liu, Z. (1994) Optimal Unit Commitment Considering Unit's Ramp-Rate Limits. Power System Technology, 18, 11-16. 
Submit or recommend next manuscript to SCIRP and we will provide best service for you:

Accepting pre-submission inquiries through Email, Facebook, LinkedIn, Twitter, etc. A wide selection of journals (inclusive of 9 subjects, more than 200 journals)

Providing 24-hour high-quality service

User-friendly online submission system

Fair and swift peer-review system

Efficient typesetting and proofreading procedure

Display of the result of downloads and visits, as well as the number of cited articles Maximum dissemination of your research work

Submit your manuscript at: http://papersubmission.scirp.org/

Or contact epe@scirp.org 